Maziarz Barbara, Makarewicz Magdalena, Kubicka Magdalena, Lapa Katarzyna, Nieradko-Iwanicka Barbara. Health problem resulting from the use of e - cigarettes. Journal of Education, Health and Sport. 2020;10(3):112-119. eISSN 2391-8306. DOI http://dx.doi.org/10.12775/JEHS.2020.10.03.011

https://apcz.umk.pl/czasopisma/index.php/JEHS/article/view/JEHS.2020.10.03.011

https://zenodo.org/record/3726425

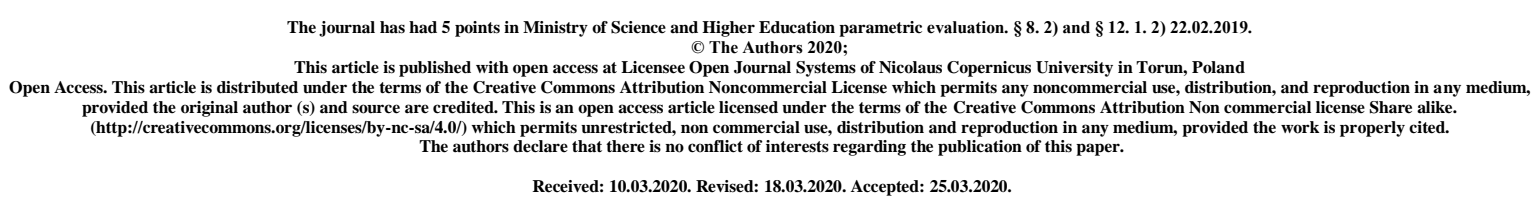

\title{
Health problem resulting from the use of e - cigarettes
}

\section{Barbara Maziarz ${ }^{1}$, Magdalena Makarewicz ${ }^{1}$, Magdalena Kubicka ${ }^{1}$, Katarzyna Lapa ${ }^{1}$, Barbara Nieradko-Iwanicka ${ }^{2}$}

${ }^{1}$ Students' Scientific Association at The Chair and Department of Hygiene, Medical University of Lublin

${ }^{2}$ Chair and Department of Hygiene, Medical University of Lublin, Radziwillowska 11Street

\begin{abstract}
Introduction: Electric smoking devices called e-cigarettes are gaining more and more popularity nowadays and vaping is becoming more and more fashionable. However their use contributes to the emergence of new health problems like lipoid pneumonia associated with vaporization.

Purpose: The purpose of the work is to analyze the advantages and disadvantages of e-cigarettes and to assess the risk of their use compared to traditional forms of smoking.

Method: The article is a review of recent publicatopns available in the PubMed database. It was searched on January 20th 2020 with key words 'e-cigarettes', 'vaping', 'health problems'. After analyzing several dozen abstracts, 18 full-text publications were selected for review.

Summary: In conclusion: vaping carries the risk of thermal injury and chemical burns caused by damage of the device, it leads to an increase in the number of vaping-addicted youth, and can also cause shortness of breath, chest pain, cough, hemoptysis, nausea, vomiting, diarrhea, abdominal pain, chills, weight loss, fatigue, malaise, headache, fever, tachycardia, tachypnoe, hypoxemia, low white blood cel count, lipoid pneumonia and pneumothorax in users.
\end{abstract}

Key words: e-cigarettes, vaping, health problems 


\section{Introduction}

Electric smoking devices called e-cigarettes are gaining more and more popularity nowadays and vaping is becoming more and more fashionable. Manufacturers ensure that e-cigarettes are less harmful than regular cigarettes, do not have tar, are convenient to use, are available in many flavours. The devices are advertised to smokers as an aid helping in smoking cessation. However e-cigarettes have many disadvantages. E-cigarette liquids contain a variety of chemical components that can have adverse effects on health. The most important declared ingredients of nicotine-based e-cigarettes include propylene glycol and glycerin, in addition to nicotine. The identified impurities include polycyclic aromatic hydrocarbons, nitrosamines, volatile organic chemicals and inorganic chemicals such as toxic metals. Endotoxins and aromatics such as diacetyl and 2,3-pentanedione have also been detected. The use of e-cigarettes contributes to the emergence of new health problems like lung disease associated with vaporization. Available data suggest that the increase in the number of patients with severe lung disease is relatively new. Although the final cause of this cluster remains unknown, the severity of the disease and the recent increase in the incidence of lipod pneumonia indicate that these cases constitute a new or newly diagnosed and disturbing cluster of lung diseases associated with vaporization.

\section{The aim}

The purpose of the work is to analyze the advantages and disadvantages of e-cigarettes and to assess the risk of their use compared to traditional forms of smoking.

\section{Materials and methods}

The article is a review of recent publicatopns available in the PubMed database. It was searched on January 20th 2020 with key words 'e-cigarettes', 'vaping', 'health problems'. After analyzing several dozen abstracts, 18 full-text publications were selected for review.

\section{Results and discussion}

First the e-cigarette was classified as a pharmaceutical and drug delivery product, but it was opposed by the courts and the Food and Drug Administration (FDA). In 2009, the Family Smoking Prevention and Tobacco Control Act (FSPTCA)underlined that it was a product containing tobacco derived nicotine and has no therapeutic properties, so it must be classified as a tobacco product[1].

Nicotine is an alkaloid antagonist of acetylcholine receptors in the peripheral and central nervous system. Cigarettes contain 1-2mg of nicotine, and the lethal dose for a child is $10 \mathrm{mg}$ it's about half a packet). Nicotine reaches the brain in a few seconds and is highly addictive. An e-cigarette has a cartridge containing nicotine $(20 \mathrm{mg})$ dissolved in humectant, which is usually propylene glycol. The construction also consists of batteries, heating elements and a switch, enclosed in a tube with a mouthpiece. These constructions are also used for smoking marijuana hash oil which is not regulated by law. The FDA is trying to tighten e-cigarettes regulations because of their danger, but users oppose it, arguing that this could result in more returns to smoking.

Some experts say that e-cigarettes can be a revolutionary tool for changing habits that can improve public health by reducing exposure to conventional cigarette smoke and reducing the possibility of addiction[2].

The study conducted by Hajek et al. compared the effectiveness of smoking cessation and side effects of e-cigarettes and nicotine-containing agents. A total of 886 middle-aged volunteers participated in the study. The groups chose their preferred method (e-cigarette or nicotine-containing agents, e.g. patches) but could not use both methods at the same time. Annual abstinence was obtained by $18 \%$ 
using an e-cigarette and $9.9 \%$ using nicotine-containing agents. Oral and throat irritation in $65.3 \%$ vs. $51.2 \%$, but vomiting was more common in people using nicotine drugs $31.3 \%$ vs. $37.9 \%$. More people using e-cigarettes stopped having problems with cough. The problem with short breath remained the same in both groups. E-cigarettes were used after the end of the study by $80 \%$ of respondents and nicotine products only by $9 \%$. Users of e-cigarettes reported less irritability than the second group[3]. A research conducted in the US in turn clearly showed that the use of e-cigarettes was not significantly associated with increased smoking cessation[4].

An interesting study was conducted on the possible relationship between the use of e-cigarettes and the increase in alcohol consumption[5]. Cross-sectional study of adults ( $\geq 16$ years) (nicotine replcement therapy-NRT) in England included 961 people who quit using e-cigarettes $(\mathrm{n}=425)$, nicotine substitutes $(n=116)$ or without assistance $(n=421)$ during the last year and still were abstainers. Former smokers who used e-cigarettes consumed about 1.69 times more units of alcohol per week compared to those who quit smoking on their own, but their alcohol consumption was similar to those who quit NRT. Data regarding differences in high-risk drinking were inconclusive. The study did not include people who used prescription drugs because these people were admonished by doctors to reduce alcohol consumption[5].

The use of e-cigarettes also causes many other dangers, such as the risk of an explosion and serious injury. Brownson described the case of a 17-year-old boy who had a crash while using an e-cigarette. The victim felt pain and swollen jaw. The patient's condition was stable, without respiratory failure. $\mathrm{He}$ had a round chin puncture, extensive lacerations in the mouth, damage to the lower incisors and damage to the left side of the jaw. Computed tomography showed a fragmented and dislocated fracture of the mandible with damage to the left central and lateral incisors. The patient underwent open fracture surgery, teeth extraction and tissue cleansing. During the 6-week period the patient recovered[6].

Electronic cigarettes have been reported to be associated with secondary burns to device or battery explosions or contact with overheating leading to burns, contact or chemical burns. In addition, soft tissue and bone injuries have been reported with or without burns. Using collective evidence, Jones et al. summarized all reported electronic cigarettes-related burns and their impact on immediate treatment, with particular emphasis on surgical treatment [7]. The most common type of burn was flame. However, there have been reports of chemical burns related to EC. The average total body surface area affected (TBSA) was $4.9 \%$ (range 1-27.25\%), with most burns partially mixed and full thickness. 22 patients underwent resection and autologous skin transplantation within 3 to 21 days. One patient had full-thickness and closed contact excision, one patient received xenograft after wound cleansing, and the other had a biosynthetic dressing on the skin. 42 patients were treated conservatively with dressings or ointments[7].

Recently, there has also been a rapid increase in the number of cases of severe lung disease associated with the use of e-cigarettes. Patients have dyspnoea, cough, chest pain and gastrointestinal symptoms such as nausea, vomiting, diarrhea and abdominal pain. The pathological cause of these cases of lung diseases remains unknown. Possible causes include: aerosolization of liquids from e-cigarettes, counterfeiting of devices with tetrahydrocannabinol (THC) or vitamin E oils, and the use of black market products. Given the growing popularity and widespread use of e-cigarettes, which are described as a safer alternative to traditional cigarettes, these cases pose a threat to public health. Hswen et al. assessed the number of cases of lung disease in people using e-cigarettes in the USA. In their report, they took into account the period from August 28, 2019, where 119 confirmed and suspected cases of lung-related lung diseases were detected until September 20, 2019, when 908-495 confirmed and 413 suspects were identified. The report may indicate an epidemic is emerging[8]. 
In another study, the authors Butt et al. analyzed lung biopsies of 17 patients. All the patients had a history of vaping marijuana or cannabis oils and clinically presented a picture of vaping-related lung damage. The lesion picture was acute or subacute in all cases with bilateral clouding of the lungs. Histopathological findings showed acute lung damage, including acute fibrous pneumonia and diffuse alveolar damage. Although the results of the histological examination were not specific, the image of foamed macrophages and vacuolization of pneumocytes was visible in all cases and may be useful diagnostic guidelines. In two cases, bronchoalveolar lavage fluid was available and contained abundant foamed macrophages. Despite glucocorticoid treatment and supportive care, two patients with thevaping-related lung disease died. Histological changes suggest that lung damage associated with vaping is a form of chemical pneumonia, not exogenous lipid pneumonia as such[9].

Mukhopadhyaya et al. described eight their patients with lung disease associated with vaping who underwent a lung biopsy. All patients were men (19-61 years old) who had respiratory symptoms after using the e-cigarettes. All patients reported THC vaporization; two of them also vaporized nicotine. The most common symptoms were fever $(n=7 / 8$ patients), cough $(n=6 / 8)$ and shortness of breath ( $n$ $=5 / 8$ ). Most patients did not have significant medical history. Four patients were hypoxic during the study. Broad-spectrum empirical antibiotics were given in seven cases. Double-sided crackles were heard over the lung fields in two patients, and double-sided coarse rales in one patient. Their laboratory results included elevated white blood cel count $(n=6 / 8)$ with predominance of neutrophils and an increase inerytrocyte sedomentation rate as well as C-reactive protein in some patients. The infection testa were negative in seven out of eight cases. No patient had clinical or laboratory evidence of other likely etiologies. Computed tomography of the chest showed a bilateral image of milky glass in all patients. In addition, some have seen mutual consolidation. Seven people were treated with corticosteroids and one with antibiotics.

Histopathological biopsy results showed that all eight samples confirmed acute lung injury manifested as organizing pneumonia, diffuse alveolar damage (DAD), unclassifiable organization of acute lung injury, or a combination of these images. Four biopsy samples showed variable visible areas of pneumonia organization, characterized by the presence of polypoid fibroblasts (Masson bodies) in the air spaces. This was accompanied by mild interstitial chronic inflammation in three cases and focal acute inflammation in the air spaces in one case. Two biopsy samples showed DAD. In the third case, the only surgical lung biopsy in this series, DAD areas overlapped with areas of organizing pneumonia (mixed pattern of acute lung injury). Both DAD biopsy samples showed vitreous membranes (acute DAD stage) as well as follicular septum expansion by fibroblasts (DAD organizational stage). Fibrous exudates of varying degrees of organization were noted in the air spaces in six out of eight biopsy samples. Overall, interstitial chronic inflammation consisting mainly of lymphocytes has been reported in six out of eight cases. A variable number of macrophages was present in all airspaces. Similarly, interstitial lipid deposits surrounded by multinucleated giant cells - as seen in exogenous lipid pneumonia - were absent in all cases. Bronchoalveolar lavage fluid in six cases was rich in macrophages, without a foamy appearance or thick vacuoles. No granulomas or malignant cells were identified, and no histological results suggested for probable alternative etiology. The main finding of the research is that acute lung injury patterns are the most consistent histological results. Fibrin exudates in the alveoli, called by some authors acute fibrous and organizing pneumonia, are also seen focal in most cases. These findings were non-specific in terms of etiology, but helped to confirm the presence of significant lung damage. It is well known that a wide range of toxic inhalers can cause acute lung damage, so it is likely that the inhalation of a potentially toxic mix of chemicals into the lungs ('vaping' and 'dabbing') can cause such injury patterns. Because electronic cigarette sprays can reach high temperatures, it is also possible that thermal injury may play a role in some patients. Finally, the fact that all patients and many in the reported literature report vaping products containing THC creates the possibility that this compound might be involved in the development of lung disease. 
Lung biopsy results described in the above cases were not specific for vaping. Both DAD and organizing pneumonia have a long list of potential etiologies that can rarely be diagnosed based on histological findings. The results of the conducted research strongly suggest that vaping does not cause true exogenous lipid pneumonia [10].

In July 2019, the Wisconsin Department of Health Services (WDHS) and the Illinois Department of Public Health (IDPH) received many reports of unclear lung disease that may have been associated with the use of e-cigarettes and related products, resulting in a coordinated public health investigation. As of August 27, 2019, a total of 53 cases met the established case definitions were reported by clinicians in Wisconsin (28 cases) and Illinois ( 25 cases). Similar cases have been reported in at least 25 states, and the Center for Disease Control and Prevention (CDC) coordinates public health responses in many states[11]. Symptoms presented by patients were: shortness of breath (46/53), chest pain (29/53), cough (44/53), hemoptysis (6/53), nausea (37/53), vomiting (35/53), diarrhea (23/53), abdominal pain (23/53), subjective fever (43/53), chills (31/53), weight loss (14/53), fatigue and malaise (24/53), headache (21/53), fever> 38oC (15/51), tachycardia> $100 / \mathrm{min}(34 / 53)$, tachypnoe> 20 breaths / $\min (22 / 51)$, saturation> 95\% (16/52) , 89-94\% (20/52), <89\% (16/52), leukopenia (42/52), abnormal chest X-ray (48/53), abnormal chest CT (48/48). The median duration of symptoms before presentation in hospital was 6 days, with a wide range (from 0 to 61 days) of duration of symptoms; $72 \%$ of patients reported within 7 days of symptom onset. A total of $72 \%$ of hospitalized patients were hospitalized in outpatient facilities (emergency depertments-ED, urgent clinics or other outpatient clinics) prior to admission due to related symptoms, and $45 \%$ of all patients who were observed on an outpatient or ED basis received antibiotics for presumptive respiratory tract infections, mainly oral azithromycin (in 12 out of 24 patients who received outpatient antibiotics) or levofloxacin (in 4). All patients who received antibiotics on an outpatient basis reported a progress in respiratory symptoms, prompting later admission to hospital.

All but three patients were hospitalized (50 patients [94\%]) and the median duration of hospital stay was 6 days. Admission to the intensive care unit due to respiratory failure was frequent (58\% of all patients; $62 \%$ of hospitalized patients), and $32 \%$ of all patients underwent intubation and mechanical ventilation (34\% of hospitalized patients). No patient received a tracheostomy[11].

In July and August 2019, five patients with acute lung injury potentially associated with e-cigarette use were identified in two hospitals in North Carolina. Patients were 18-35 years of age and all experienced worsening of dyspnoea, nausea, vomiting, abdominal discomfort and fever. All patients showed tachypnea with increased respiration during the examination, hypoxemia and bilateral pulmonary infiltrates on the X-ray of the chest. All patients have recently used cannabis oils or concentrates in e-cigarettes. At admission, all patients had an elevated white blood cell count with a predominance of neutrophils and a lack of eosinophilia. Initially, they were empirically treated with antibiotics for putative community-acquired or aspirative pneumonia, but all developed worsening respiratory failure within 48 hours of admission. Blood and sputum cultures were negative for bacterial pathogens; tests for influenza, Mycoplasma and Legionella were also negative. Computed tomography of the chest revealed diffuse infiltrates with the predominance of "milky glass" and nodal infiltrates in all patients. Three patients underwent bronchoscopy with bronchoalveolar lavage on days 3-5 in a hospital, resulting in a combination of neutrophils, lymphocytes and macrophages filled with vacuole, but with no evidence of follicular hemorrhage or eosinophilia. The cytology of the wash was stained with red oil, which was confirmed by extensive lipid in follicular macrophages. A diagnosis of acute exogenous lipoid pneumonia was made in all five patients. They all improved clinically within 24-72 hours after starting intravenous methylprednisone (120 mg - $500 \mathrm{mg}$ daily).

One potential explanation for acute lipoid pneumonia among these patients is that aerosol oils inhaled from e-cigarettes build up in distal airways and alveoli and cause a local inflammatory response that 
interferes with gas exchange. Lipoid pneumonia has long been described by sucking oil into the lungs and has been associated with the use of e-cigarettes in some case reports. Symptoms of lipoid pneumonia are often nonspecific with variable chest imaging, which can lead to delayed or incorrect diagnosis[12]

Vaping was the most likely cause of recurrent pneumothorax as in an 18-year-old Caucasian man described by Bonilla et al.. The patient came to the emergency department in January 2019 because of sudden pain on the right side of the chest during sleep. He denied the occurrence of excessive cough, recent respiratory tract infection, or pre-pain trauma. He reported many episodes of daily vaping and occasional marijuana use, but he did not smoke or use smokeless tobacco. Chest x-ray showed a large right-sided pneumothorax. A suction tube was placed in the chest. During hospitalization he received a patch of lidocaine every 24 hours, acetaminophen $(650 \mathrm{mg})$ every 6 hours and ketorolac $(15 \mathrm{mg})$ every 6 hours as needed. Pneumothorax resolved over the next few days, and the tube was removed gradually. One week after discharge from the hospital, the patient returned for sudden chest pain and shortness of breath. After previous discharge, he used e-cigarettes daily, but there was no fever, chills, hemoptysis, cough, upper respiratory tract infection, trauma or recent air travel. During the physical examination his vital signs were normal. X-ray examination revealed the presence of large recurrent spontaneous pneumothorax on the right without mediastinal displacement. Another CT scan of the chest without contrast after fitting the thoracic tube showed a small pneumothorax with residual subsegmental atelectasis and small vesicles of the right limb on the right side. Spontaneous pneumothorax was classified as secondary. The patient was treated with a tube. During the second admission, our patient was treated with a lidocaine patch every 24 hours, acetaminophen $(650 \mathrm{mg})$ every 6 hours as needed and ibuprofen $(600 \mathrm{mg})$ every 6 hours as needed. Patient observation 2 weeks after removing the tube from the chest confirmed the disappearance of pneumothorax. During the follow-up visit, he reported that he had stopped using all vaping products[13]. The described case is the first regarding the correlation between vaping and recurrent spontaneous pneumothorax. It is thought that marijuana smokers have an increased risk of spontaneous pneumothorax being partly associated with deep inhalation followed by the Valsalva maneuver during exhalation. Repeated deep inhalation by a highly resistant device, causing a Muller maneuver and generating high negative intrachest pressure, has also been proposed as a mechanism of spontaneous pneumothorax in marijuana smokers. Given the airflow resistance of capsule-based electronic cigarettes, such as those used by this patient, there is certainly the possibility of spontaneous pneumothorax.

The 2018 National Academies of Sciences, Engineering and Medicine showed significant evidence that young people who get into vaping smoke cigarettes more often than those who don't use ecigarettes [14] The rapid increase in the use of electronic cigarettes among young people over the past decade has become a serious public health problem, especially as it is related to the recent increased incidence of lung diseases. In the years 2017-2019, a record increase in the popularity of e-cigarettes among teenagers was recorded. Monitoring the Future conducted surveys for the twelfth, tenth and eighth grade students every year. The survey covered 43,703 respondents in 2017, 44,482 in 2018 and 42531 in 2019 . The overall response rate was $80 \%$ for the 12 th grade, $86 \%$ for the 10 th grade and $88 \%$ for the 8th grade, with no $100 \%$ attendance due to the students' absence. The current activities of the ecigarette industry, government agencies and schools have so far proved to be insufficient to stop the rapidly growing popularity of vaping among teenagers. Particularly worrying is the accompanying increase in the proportion of adolescents who are physically addicted to nicotine. New solutions are sought to protect young people from nicotine addiction when the developing brain is particularly susceptible to its harmful effects [14].

In another Monitoring the Future study, in which he examined random groups of students in classes 12,10 and 8 , the surveys covered 13,850 respondents.As in other studies, it showed a rapid increase in 
the use of e-cigarettes among students - 10.0 percentage points among 12-graders, 7.9 percentage points among 10-graders and 2.6 percentage points among 8-graders. The use of nicotine with any product increased significantly, by 5.2 percentage points from $23.7 \%$ to $28.9 \%$, in a sample of 12 graders. This increase was due to the use of e-cigarettes, as the use of each of the remaining 6 nicotinecontaining products slightly decreased [15].

In Colorado, in 2017, according to the national youth risk behavior study (YRBS), one in four students in grades 9 to 12 reported vaping, which is twice the national average.The Colorado Department of Public Health and the Environment conducted a larger, state-specific survey among young people. The Healthy Kids Colorado Survey is conducted every two years among separate, randomly selected groups of junior high school and high school students, using methods consistent with those used in YRBS. 4774 students from 157 public secondary schools in this state participated in the 2017 study. Among students in all four classes, $27 \%$ reported the use of an electronic device to inhale nonmarijuana fumes in the last 30 days (compared with $26 \%$ in 2015). The popularity of e-cigarettes increased in the upper classes of $18.5 \%$ reporting use in 9th grade, $25.3 \%$ in 10th grade, $31.7 \%$ in 11th grade and $33.6 \%$ in 12th grade. Colorado State developed the Health Act, recommending that health care professionals check their youth not only for smoking but also for e-cigarettes. There is also information on the effects of nicotine on health. There is also free counseling for young people trying to get out of addiction. He also recommends screening for other risk behaviors and providing appropriate advice [16].

Currently, 'Pod Mod' is entering the market - small, charged devices that spray liquid solutions containing nicotine, aromas and other contents enclosed in cartridges. According to Nielsen, as of January 27, 2018, Juul's popular brand won $49.6 \%$ of the e-cigarette market. Devices of this brand contain nicotine concentrations from 2 to 10 times higher than in most e-cigarette products. Juul's website says that there is $0.7 \mathrm{ml}$ nicotine per capsule (concentration, $59 \mathrm{mg}$ per milliliter [5\%]) equivalent to about 20 flammable cigarettes.Juul needs less energy to provide high doses of nicotine. The devices measure $9.4 \mathrm{~cm}$ by $1.5 \mathrm{~cm}$ by $0.8 \mathrm{~cm}$ and weigh $0.01 \mathrm{~kg}$ and resemble USB drives. In addition. The 'Pod Mod' give no unpleasant taste in the mouth. The e-cigarette aerosol may contain metals, volatile organic compounds and flavorings that can be harmful when inhaled, especially for adolescents who actually report breathing symptoms more often than non-users [17].

There were animal studies conducted to evaluate the health effects of vaping. The prototype electronic cigarette and three formulations were evaluated in a 90-day inhalation study in rats followed by a $42-$ day recovery period. Animals were randomly assigned to groups for exposure to low, medium and high doses of vehicle aerosols (a mixture of glycerol and propylene glycol); carrier and $2.0 \%$ nicotine; or carrier, $2.0 \%$ nicotine and flavoring.Dose-related decreases in thymus and spleen mass and increases in BALF lactate dehydrogenase, total protein, alveolar macrophages, neutrophils and lung mass have been observed. Histopathological assessments revealed sporadic increase in nasal segment 1-4 epithelial hypertrophy and vacuolization. After the recovery period, the effects on the nose and BALF persisted, while other effects subsided. In addition, rats exposed to high doses had lower body weight and smaller increases for each formulation tested, and males tended to proportionally greater decreases than females. In addition, they showed a reduction in food consumption[18] .

\section{Conclusions}

The reviewed articles provide evidence that electronic cigarettes are not a safe alternative to traditional cigarettes. It is doubtful if their use could help in nicotine addiction. Vaping carries the risk of thermal injury and chemical burns caused by damage of the device, it leads to an increase in the number of vaping- addicted youth, and can also cause shortness of breath, chest pain, cough, hemoptysis, nausea, vomiting, diarrhea, abdominal pain, chills, weight loss, fatigue , malaise, headache, fever, tachycardia, tachypnoe, hypoxemia, low white blood cel count, lipoid pneumonia and pneumothorax in users. 


\section{References:}

1. Cobb NK, Abrams DB. E-cigarettes or Drug- Delivery Device? Regulating Novel Nicotine Producs, N Engl J Med. 2011;365(3):193-5.

2. Etter JF, Bullen C. A longitudinal study of electronic cigarette users. Addict Behav. 2014; 39 : 491-4.

3. Hajek P, Phillips-Waller A, Przulj D, Pesola F, Myers Smith K, A Randomized Trial of ECigarettes versus Nicotine-Replacement Therapy N Engl J Med. 2019 ;380(7):629-637

4. Patil S., Arakeri G., Patil S., Baeshen H. Are electronic nicotine delivery systems (ENDs) helping cigarette smokers quit? - A current evidence, Journal of Oral Pathology \& Medicine, 2019; 00: 1-9.

5. Jackson S, Beard E, Michie S, West R, Brown J, Is the use of e-cigarettes for smoking cessation associated with alcohol consumption? A population-level survey of successful quitters in England, Addictive Behaviors, 2020;101: 106-138.

6. Brownson EG, Thompson CM, Goldsberry S, Chong HJ, JB Friedrich, Pham TN, Arbabi S,Carrougher GJ, Gibran NS Explosion Injuries from E-Cigarettes. N Engl J Med 2016; 375:1400-1402,DOI: 10.1056/NEJMc1608478.

7. Jones CD, Ho W, Gunn E, Widdowson D, Bahia H.E-cigarette burn injuries: Comprehensive review and management guidelines proposal. Burns.2019;45(4): 763-771.

8. Hswen Y, Brownstein J, Real-Time Digital Surveillance of Vaping-Induced Pulmonary Disease, N Engl J Med. 2019, 381:1778-1780.

9. Butt YM, Smith ML, Tazelaar HD. Pathology of Vaping-Associated Lung Injury, N Engl J Med; 2019, 381:1780-1781.

10. Mukhopadhyay S, Mehrad M, Dammert P. Lung Biopsy Findings in Severe Pulmonary Illness Associated With E-Cigarette Use (Vaping): A Report of Eight Cases, Am J Clin Pathol.2019; 153: 30-39.

11. Layden JE, Ghinai I, Pray I, Kimball A, Layer M, Tenforde MW, Navon L, Hoots B,Salvatore PP, Elderbrook M, Haupt T, Kanne J, et al. Pulmonary Illness Related to E-Cigarette Use in Illinois and Wisconsin — Preliminary Report. N Engl J Med 2020; 382:903-916.

12. Davidson K, Brancato A, Heetderks P, Outbreak of Electronic-Cigarette-Associated Acute Lipoid Pneumonia - North Carolina, July-August 2019, MMWR Morb Mortal Wkly Rep. 2019 ; 68(36): 784-786.

13. Bonilla A, Blair A, Alamro S. Recurrent spontaneous pneumothoraces and vaping in an 18year-old man: a case report and review of the literature, Journal of Medical Case Reports. 2019; 283 (2019) doi:10.1186/s13256-019-2215-4.

14. Colorado Department of Health and Environment. Colorado Vaping-Associated Illness Outbreak Report. https://www.colorado.gov/pacific/cdphe/vaping-lung-illness cited on March $\underline{\text { 8th } 2020 .}$.

15. Patrick ME, O'Malley $M$ et al. Trends in Reported Marijuana Vaping Among US Adolescents, 2017-2019. JAMA. 2020;323(5):475-476.

16. Miech R, Johnston L, O’Malley PM, Bachman JG. Adolescent Vaping and Nicotine Use in 2017-2018 - U.S. National Estimates. N Engl J Med 2019; 380:192-193 DOI: $10.1056 / \mathrm{NEJMc} 1814130$

17. Barrington-Trimis JL, Leventhal AM. Adolescents' Use of "Pod Mod" E-Cigarettes — Urgent Concerns. N Engl J Med 2018;379:1099-1102.

18. Werley M., Kirkpatrick D., Oldham M., Jerome A. Toxicological assessment of a prototype ecigaret device and three flavor formulations: a 90-day inhalation study in rats. Inhal Toxicol. 2016 ; 28(1): 22-38. 